\title{
Aus den Berliner Gesellschaften. Sitzung·der Ophthalmologischen Gesellschaft
}

vom 28. II. 1901

II err Fehr stellt zwei Fälle von Keratitis difîusa (schwere Form) vor. Beide Patienten waren infolge von Labyrinthaffection auch taub.

Sitzungsbericlite.

397

Herr Prof. Krönig a. G.: Ueber Lumbalpunction.

Der Vortragende rnacbt zunäcbst eine kurze anatomische Beinerkung. Die beiden

Subduralräume communiciren nicht miteinander, wohl aber dia Subarachnoidealräume des

Gehirns und Rückenmarks. Die Versuche Quincke's mit Zinnober- Injection von unten ergaben schon nach $\beta-12$ Stunden an der Basis beim Eintritt des Opticus in das Auge positives Resultat in der Opticusscheide. In den Ventrikeln fand sich niemals der Zinnober; ebenso gelang der Versuch nicht beim Cadaver. Der physiologische Strom besteht von oben herab und von unten hinauf. Die Versuche ergaben ferner, dass der Druck verschieden hoch ist.

Nach Krönig's Ansicht hat jedoch Quinoke, olme es zu wollen, i miner auch aspirirt und dabei ca. 8 com Flüssigkeit verloren. Diesen 1.'ehler suchte Krönig auszuschalten durch einen besonderen Heber-apparat zum Messen der Flüssigkeit; ausserdem wies er darauf hin, wie verschieden oft der Druck im Liegen resp. Sitzen ist (120 resp. $420 \mathrm{~mm}$ ). Sehr in Betracht kommen die pulsatorischen Schwankungen bei der Fortpflanzung; fehlen diese, so ist jedes Ablassen von Flüssigkeit mit Gefahr verbunden, sind sie vorhanden, so kann man viel ablassen. Normaler Weise betragen sie $2-5 \mathrm{~mm}$.

Die Stelle des Einstichs zum Zwecke der Lumbalpunction ist am besten im V. Sacralraum (nicht im III. und. IV.), 4 - $5 \mathrm{~cm}$ unter der Cauda equina. - Vortragender erwähnt auch Manz's Mittheilung über Hydrops der Opticusscheide ohne Stauungspapille. Er sagt, dass mit < ler Punction früher Erhöhung des Drucks nachzuweisen sei als mit deni Ophthalmoscop; in der normalen Flüssigkeit finden sich fast gar keine morphologischen Elemente. Auch die Differential-Diagnose: Tumor oder Meningitis, sei mittels Punction leicht zu stellen.

Dis cus sion:

Herr Schuster a. G. hat bei Tumoren nach Punction die Stauungspapille oft nicht zurückgehen sehen; nur in 2 Fallen trat Erfolg ein. Er liess sogar Mengen von 40 - 50 ceni Flüssigkeit ah. Herr Krönig hat keine Erfahrung darüber, wie oft die Stauungspapille zurückgeht. Schlechte Ergebnisse schiebt Krönig meist dem Operateur zu.

Herr Hirschberg fragt an. ob die Kopfschmerzen bei Tumoren dureh Function gelindert werden. Herr Krönig berichtet von einem Fall, in welchem Kopfschmerzen und Erbrechen auf $\Lambda$ Vochen sistirten. Die Patientin verlangte stets wieder nach der Punction.

Herr Ginsberg macht auf die Verschiedenheit in der Beziehung von Oedem und Stauungspapille aufnierksam. 
Herr Steindorff demonstrirt ein mikroskopisches Präparat von einem Papillom der Conjunctiva mit sehr geringem Bindegewebe und starker Epithelwucherung, ferner zeigt er eine verkalkte grosse Linse.

Pollack.

2Tí.

: 